\title{
Maternal Clofibrate Administration Amplifies Fetal Peroxisomes ${ }^{1}$
}

\author{
GOLDER N. WILSON, TERESA KING, J. CRAIG ARGYLE, AND RAFAEL F. GARCIA \\ Departments of Pediatrics and Pathology, University of Texas, Southwestern Medical Center, \\ Dallas, Texas 75235
}

\begin{abstract}
To study the effects of peroxisome proliferators on fetal biogenesis, $400 \mathrm{mg} / \mathrm{kg}$ oral clofibrate was administered to pregnant mice beginning at $\mathbf{d} 6$ of gestation. Maternal/fetal tissues from three separate experiments were harvested between gestational d 13 and 19 and maternal/fetal tissues were assayed for peroxisomal matrix, membrane-associated, and integral membrane proteins. By comparison to control pregnancies that received vehicle only, clofibrate produced a 4 - to 5-fold increase in the levels of peroxisomal membrane protein 70 , a 1.5- to 2fold increase of dihydroxyacetone phosphate acyltransferase (DHAP-AT) sp act, and a 1.2- to 1.8-fold increase of catalase sp act in fetal liver of $19 \mathrm{~d}$ gestation. Fetal liver endoplasmic reticulum and peroxisomes were also amplified as visualized by electron microscopy. Clofibrate exhibited maximal effects on maternal tissues by $6 \mathrm{~d}$ of oral treatment with increases in peroxisomal membrane protein 70 occurring most clearly in maternal liver; DHAP-AT activity was increased in maternal liver, kidney, and brain but not in lung. Slight increases in DHAP-AT activities were evident in fetal brain, lung, and placenta as compared with the greater increases in liver and kidney. There was a general increase in peroxisomal proteins between 13 and 19 gestational $d$ in all fetal tissues except placenta, and the effect of clofibrate was evident by gestational d 13 in lung and placenta. Minimal changes in the activities of acid phosphatase (lysosomal enzyme) or glycerol-3-phosphate acyltransferase (microsomal enzyme) were observed in maternal or fetal tissues although the latter enzyme was increased $10-30 \%$ by clofibrate in maternal brain, fetal lung, and placenta. The degree of amplification peroxisomal membrane portion $70>$ DHAP-AT $>$ catalase observed in several tissues after clofibrate treatment is consistent with a similar order in the sequence of peroxisomal assembly. The ability to manipulate fetal peroxisomes by maternal pharmacology provides a potentially useful system for studying the regulation of peroxisomal biogenesis. (Pediatr Res 29: 256-262, 1991)
\end{abstract}

\section{Abbreviations}

PMP, peroxisomal membrane protein DHAP-AT, dihydroxyacetone phosphate acyltransferase GP-AT, glycerol-3-phosphate acyltransferase $N_{G}$, Nycodenz gradient fraction

Received July 12,1990 ; accepted October 17, 1990.

Correspondence and reprint requests: Golder N. Wilson M.D., Ph.D., Depart ment of Pediatrics. University of Texas Southwestern Medical Center, 5323 Harry Hines Blvd., Dallas. TX 75235.

Supported in part by the Biological Humanics Foundation, Dallas, TX.

' Presented in part at the Annual Meeting of the Society for Pediatric Research/ American Pediatric Society. Anaheim, CA. May 7-11. 1990
Recent studies of human peroxisomal deficiency disorders have revealed that a dissociation may occur between the amounts of peroxisomal matrix proteins and those integral to the membrane $(1,2)$. Virtually no normal peroxisomes are visible in Zellweger syndrome fibroblasts because matrix proteins such as catalase are absent or relocated in the cytoplasm. Membraneassociated proteins such as DHAP-AT are completely deficient, perhaps because of degradation (3). By contrast, proteins integral to the peroxisomal membrane (PMP) demonstrate amounts comparable to those of control cells.

Peroxisomal membranes are present in cells as "ghosts" with expanded and empty centers indicating sequential assembly and/ or independent regulation of matrix components (1). Because peroxisomal biogenesis is assumed to occur by budding from preexisting peroxisomes (4), membrane extension may occur first followed by organization of the internal milieu. Study of peroxisomal assembly with attention to matrix, membrane-associated, and integral membrane components will be required to understand biogenesis and its consequences for peroxisomal function and disease.

Ontogeny provides a natural system in which to examine the biogenesis of peroxisomes. The synthesis of membrane and matrix components may be integrated quite differently in separate tissues, judging by the 10 -fold greater volumes of liver or kidney peroxisomes compared with the "microperoxisomes" of connective tissue or brain (3). Although fetal peroxisomes would presumably derive from those in the egg, there have been few studies documenting their ontogeny. It is surprising that peroxisomes were not evident in rat liver before $14-15 \mathrm{~d}$ of gestation but are abundant by $\mathrm{d} 19(5,6)$. Perhaps membrane components predominate before that time, with matrix components being synthesized later in development. Briere (7) identified peroxisomes in fetal kidney, and they are present in human chorionic villi at about 10 wk gestation (8). The few studies available are consistent with a gradual increase of peroxisomes in most tissues as mammalian development progresses, with the highest activities of peroxisomal enzymes in liver and kidney $(9,10)$. This pattern continues during postnatal life in rats, with activities of peroxisomal $\beta$-oxidation enzymes peaking at birth in liver and at about $70 \mathrm{~d}$ in heart (11). No studies have examined the coordinated ontogeny of peroxisomal membrane and matrix proteins as reported here.

Additional understanding of biogenesis might be provided if peroxisomal ontogeny could be manipulated by administering peroxisome proliferators such as clofibrate or phthalate ester plasticizers (10). These agents in fact produce a pleiotypic response with proliferation of peroxisomes and other organellar components such as the endoplasmic reticulum. Chronic administration to rats also results in a high incidence of hepatocarcinoma (10). Intriguing aspects of the proliferation reaction include species specificity (12) and the inductive ability of certain peroxisomal substrates (13). Clofibrate is active as a peroxisome proliferator in rodents, but has not been useful as a treatment for human peroxisomal deficiency disorders (14). In rats, selec- 
tive amplification of peroxisomal components is demonstrated, with certain enzymes involved in peroxisomal $\beta$-oxidation increasing 20 - to 30 -fold, whereas catalase increases only 2 -fold. These increases in enzyme activity appear to occur at the transcriptional level, based on studies with cDNA probes for acyl $\mathrm{CoA}$ oxidase and bifunctional enzyme (15). Despite an intensive search for binding proteins that might mediate the proliferative response, the mechanism of proliferation is still unknown (16).

Only one group has examined the ability of maternal clofibrate treatment to induce fetal peroxisome proliferation $(17,18)$. An increase in rat liver peroxisome number and fatty acid oxidation enzymes suggested that fetal proliferation occurred. Here we confirm this observation in mice and examine the effect of maternal clofibrate on the matrix, membrane-associated, and integral membrane proteins of fetal peroxisomes.

\section{MATERIALS AND METHODS}

Materials. Nycodenz was obtained as the solid from Nycomed Diagnostics through Robbins Scientific (Mountain View, CA), nitrocellulose membranes from Amersham (Arlington Heights, IL), immunoblotting reagents and clofibrate from Sigma Chemical Co. (St. Louis, MO), goat anti-rabbit peroxidase-conjugated antibody from Cappel (Malvern, $\mathrm{PA}$ ), ${ }^{32} \mathrm{P}_{-} \mathrm{PO}_{4}$ from Amersham, and electrophoresis reagents from Bio-Rad (Richmond, CA). Swiss-Webster mice were obtained from Charles River Breeding Laboratories (Wilmington, MA) at 6-8 wks of age and maintained on regular food and light/dark cycles.

Animal studies. Breeding was initiated by placing one male mouse with four females overnight with $\mathrm{d} 0$ of gestation beginning the following morning. Mice were weighed daily to determine pregnancy and clofibrate treatment $(400 \mathrm{mg} / \mathrm{kg})$ initiated at $\mathrm{d} 6$ of gestation as a freshly diluted solution in peanut oil administered daily using Metafane (Pitman-Moore, Inc., Washington Crossing, $\mathrm{NJ}$ ) anesthesia and an orogastric cannula. Control mothers received equivalent volumes of peanut oil. Mice were killed by cervical dislocation at 13,15, 17, and $19 \mathrm{~d}$ gestation. Maternal (brain, lung, liver, kidney) or fetal (brain, lung, liver, kidney, placenta) tissues were dissected rapidly and immediately frozen on a slab of dry ice. Before enzyme or immunoblotting assays, tissues were thawed and suspended in $0.25 \mathrm{M}$ sucrose using a hand-held glass homogenizer. Three separate breeding experiments were performed, with tissues from all the fetuses in a given litter being combined in one homogenate. Tissues from mothers were homogenized individually. For enzymes, duplicate assays were performed on each tissue homogenate and the six values from three experiments averaged for mean and SEM. The significance of differences between clofibrate-exposed and control tissue mean values was assessed by the $t$ test $(p<0.05)$.

Enyzme assays. Peroxisomal marker enzymes included the matrix enzyme catalase, assayed by the method of Peters et al. (19), and the membrane associated enzyme DHAP-AT. DHAPAT was assayed by the method of Jones and Hajra (20) after synthesizing ${ }^{32} \mathrm{P}$-DHAP from ${ }^{32} \mathrm{P}_{-} \mathrm{PO}_{4}$ and 3-phosphoglycerate kinase/glyceraldehyde-3-phosphate dehydrogenase (BoehringerMannheim, Indianapolis, IN). Conversion of $1 \mathrm{nmol}{ }^{32} \mathrm{P}-\mathrm{DHAP}$ into lipid soluble (acylated) form per min represented 1 unit of activity. A similar assay (21) was employed for GP-AT, which is mainly localized to microsomes. The mitochondrial marker was cytochrome c oxidase assayed by the method of Leighton $e t$ al. (22). Lactate dehydrogenase assayed by the method of Racker (23) was employed as a cytoplasmic marker, and acid phosphatase assayed with nitrophenyl phosphate (Sigma kit no. 435) was a marker for lysosomes. The sp act of these enzymes were based on $\mu \mathrm{mol}$ of substrate converted per min. Protein was quantitated by the method of Bradford (24) or, in the presence of SDS, by the method of Lowry (25) after trichloroacetic acid precipitation.

Mouse peroxisome purification. Four 6- to 8-wk-old mice were killed by cervical dislocation and their livers (about $9 \mathrm{~g}$ total) diced in $27 \mathrm{~mL}$ cold $0.25 \mathrm{M}$ sucrose, $10 \mathrm{mM}$ Tris- $\mathrm{HCl} \mathrm{pH} \mathrm{7.4,}$ $1 \mathrm{mM}$ EDTA. Tissue disruption was performed in a PotterElvesham homogenizer with a smooth pestle $(50-\mathrm{mL}$ vessel) mounted on a rotary motor (1500 rpm, one pass taking $1 \mathrm{~min}$ ). Differential centrifugation was performed at $4^{\circ}$ essentially as described by Leighton et al. (22) using a Beckman J2-21 centrifuge, a type JA-20 rotor, and 50-mL polyethylene tubes. A "light mitochondrial" or $\mathrm{L}$ fraction was obtained after $l$ ) centrifuging three times for $10 \mathrm{~min}$ at $2250 \mathrm{rpm}(600 \times \mathrm{g})$ and discarding the pellet (nuclear debris); 2) centrifuging three times for $10 \mathrm{~min}$ at $5250 \mathrm{rpm}(3300 \times \mathrm{g})$ and discarding the pellet (mitochondria), and 3 ) centrifuging three times for $10 \mathrm{~min}$ at $14500 \mathrm{rpm}(25000$ $\times g$ ) and retaining the pellet. At each stage, the initial pellet was homogenized in an equivalent volume of fresh sucrose buffer and centrifuged again under the indicated conditions. Four $0.7-$ $\mathrm{mL}$ aliquots of the $\mathrm{L}$ fraction were layered over $2.5 \mathrm{~mL}$ of $27.6 \%$ Nycodenz in $3.4 \mathrm{~mL}$ polycarbonate tubes and centrifuged for 1 $\mathrm{h}$ at $75000 \mathrm{rpm}(231840 \times \mathrm{g})$ in the TLA 100.3 rotor at $4^{\circ}$. The loose pellet ( $\mathrm{N}$ fraction) was collected with residual Nycodenz after aspiration of the supernatant and placed on a Nycodenz gradient consisting of six $2-\mathrm{mL}$ shelves $(44,40,36,32,28$, and $24 \%$ ) pipetted and equilibrated overnight at $4^{\circ}$ in $12-\mathrm{mL}$ polycarbonate tubes. $\mathrm{N}$ fraction $(1 \mathrm{~mL}$ ) was overlaid (sinking about $1 \mathrm{~mL}$ into the gradient) and centrifugation was $6 \mathrm{~h}$ at 35000 $\mathrm{rpm}$ and $4^{\circ}$ in a SW 41 swinging bucket rotor. The peroxisomal band, about $4 / 5$ down the gradient, was aspirated with a needle $\left(\mathrm{N}_{\mathrm{G}}\right.$ fraction), diluted $2 / 3$ with $0.25 \mathrm{M}$ sucrose, and concentrated by centrifuging for $10 \mathrm{~min}$ at $12000 \mathrm{rpm}$ and $4^{\circ}$ in the Beckman JA-20 rotor. Membranes were purified as described (26) by diluting $0.06 \mathrm{~mL}(0.2-0.5 \mathrm{mg}$ protein) with $2.94 \mathrm{~mL}$ of $100 \mathrm{mM}$ sodium carbonate $\mathrm{pH} 11.5$, centrifuging $1 \mathrm{~h}$ at $75000 \mathrm{rpm}$ and $4^{\circ}$ in the Beckman TLA 100.3 rotor, and suspended in $300 \mu \mathrm{L}$ of normal saline.

Preparation of antibodies to mouse PMP. The peroxisomal membrane suspension was combined with $1.5 \mathrm{~mL}$ of complete Freund's adjuvant (Sigma) and divided into 20 intradermal injections along the back of each of two 20 -wk-old New Zealand White rabbits. In $2 \mathrm{wk}$, a second s.c. injection was given, which contained an equivalent amount of PMP. Serum collected 10 wk later and at 2 -wk intervals thereafter was frozen at $-70^{\circ} \mathrm{C}$ until used.

$S D S-P A G E$ and immunoblot analysis. Gradient fractions or organ homogenates were diluted $1 / 2-1 / 3$ in loading buffer and applied to $7.5 \%$ SDS-PAGE gels in a Bio-Rad MiniProtean II apparatus using protocols supplied by the manufacturer. Electrophoresis was for $0.5 \mathrm{~h}$ at $200 \mathrm{~V}(50 \mathrm{~mA})$ and protein blotting was accomplished using a BioRad Mini Trans-Blot apparatus and nitrocellulose membranes. Blocking with $5 \%$ BSA, $10 \mathrm{mM}$ Tris- $\mathrm{HCl} \mathrm{pH} \mathrm{7.4,150} \mathrm{mM} \mathrm{NaCl}$ for $18-24 \mathrm{~h}$ at $25^{\circ} \mathrm{C}$ was followed by incubation with rabbit antiserum diluted $1: 100$ in $50 \mathrm{mM}$ Tris- $\mathrm{HCl} \mathrm{pH} \mathrm{7.4,0.15} \mathrm{M} \mathrm{NaCl,} 5 \mathrm{mM}$ EDTA, $0.25 \%$ gelatin, $0.05 \%$ NP-40 (Sigma) for $1 \mathrm{~h}$ at $25^{\circ} \mathrm{C}$. Development was with goat anti-rabbit serum and the peroxidase detection kit provided by Cappel. The intensity of PMP 70 bands was qualitatively converted to $\mu \mathrm{g} / \mathrm{mg}$ of homogenate by comparison to the standard curve.

Electron microscopy. For assessment of purity, peroxisome suspensions were stored at $-70^{\circ} \mathrm{C}$, then diluted into $0.25 \%$ glutaraldehyde (Sigma) and stored at room temperature until examination. After centrifugation for $1 \mathrm{~min}$ in a microcentrifuge, the pellet was postfixed with osmium tetroxide and embedded in Epon 812. Adult and fetal mouse liver specimens were stained with the catalase substrate diaminobenzidine (18), then processed for electron microscopy as above.

\section{RESULTS}

The purification of peroxisomes from mouse liver was accomplished by modifications of prior methods for differential and Nycodenz gradient centrifugation $(22,27,28)$. The final gradient 
$\left(\mathrm{N}_{\mathrm{G}}\right)$ fraction was 25 -fold enriched in catalase $\mathrm{sp}$ act with less than $1 \%$ contamination by other organelles as shown in Figure $1 B$. Injection of sodium carbonate-treated (26) $\mathrm{N}_{\mathrm{G}}$ fraction into rabbits generated antisera that detected 145-, 70-, 60-, 55-, 36-, and $22-\mathrm{kD}$ peptides after SDS-PAGE and immunoblotting. The specificity of these antibodies for PMP is suggested by the codetection of $145-, 70-, 60-$, and $55-\mathrm{kD}$ peptides with the peroxisomal marker enzyme DHAP-AT in high-density fractions of the Nycodenz gradient (Fig. $1 A$ ). No peptides are visualized when the PMP antiserum is used for immunoblotting of purification fractions depleted in peroxisomes (data not shown). PMP 70 was the most prominent peptide detected by all antisera as illustrated by the developmental studies described below.

The proliferative effect of clofibrate on peroxisomes in both maternal and fetal tissues is demonstrated by electron microscopy and assay of peroxisomal proteins. Peroxisome density in maternal liver after $7 \mathrm{~d}$ of oral clofibrate administration (Fig. $2 B$ ) was increased almost 2-fold compared with control maternal liver (Fig. 2A). Additional experiments showed that peroxisome proliferation in maternal liver plateaued after $6 \mathrm{~d}$ of oral clofibrate administration, meaning that maximal maternal proliferation had been attained throughout the period of fetal sampling. There was also a striking proliferation of the endoplasmic reticulum and an increased number of peroxisomes (arrows) in 15-d
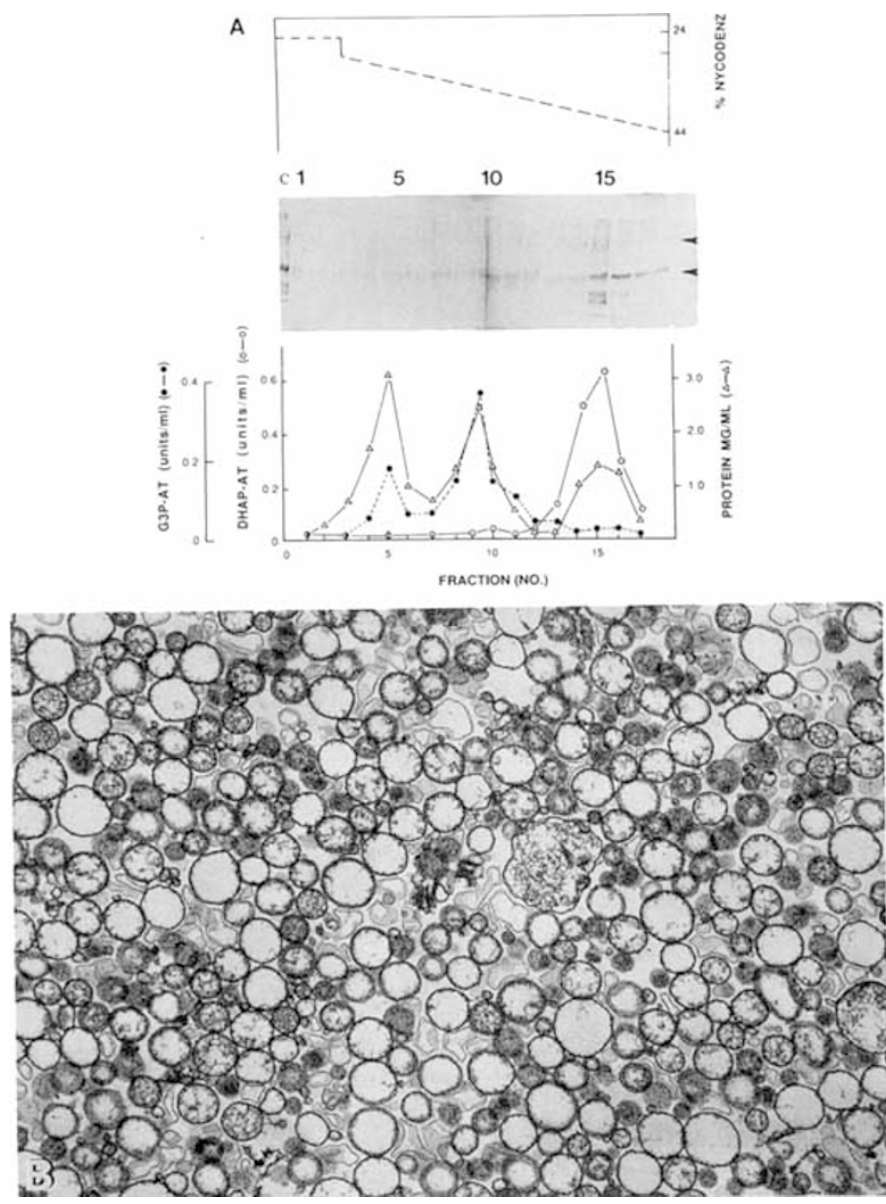

Fig. 1. Nycodenz gradient purification of mouse liver peroxisomes A. Nycodenz gradient fractions $(0.7 \mathrm{~mL})$ were assayed for GP-AT (O), DHAP-AT $(O)$, or total protein $(\triangle)$ as shown in the lower panel. The middle panel shows the immunoblot obtained after 7.5\% SDS-PAGE of $0.05 \mathrm{~mL}$ aliquots of each gradient fraction and reaction with antibody to peroxisomal membranes. Arrous indicate the positions of 145- and 70$\mathrm{kD}$ proteins as estimated from standards and lane $c$ shows a control mouse $N_{G}$ fraction for comparison. $B$, electron microscopic examination of pooled Nycodenz gradient fractions $15-17$ ( $N_{G}$ fraction). Magnification $2000 x$. fetal liver from clofibrate-treated (Fig. 2D) versus control (Fig. $2 C$ ) pregnancies. Peroxisomal density could not be reliably quantitated in fetal tissues because of relatively poor diaminobenzidine staining (Fig. $2 C$ and $D$ ).

The rabbit antiperoxisomal membrane antibody with bias toward PMP 70 allowed a comparison of this peptide in clofibrate-treated and control tissues. The amount of PMP 70 detected in immunoblotting experiments could be qualitatively estimated using the standard curve shown in the upper panel of Figure 3. Duplicate aliquots of sodium carbonate-purified membranes from the mouse $\mathrm{N}_{\mathrm{G}}$ fraction were converted to known amounts of PMP 70 by assuming equimolar composition of PMP 145, 70, 36, and 22 in the membrane as suggested by SDSPAGE gels stained with Coomasie blue or silver (29). This assumption implies that $25 \%$ of total membrane protein mass is PMP 70, allowing conversion of band intensity to $\mu \mathrm{g}$ of PMP 70 per mg of tissue homogenate loaded on the gel. Although this conversion factor may not be accurate, comparisons of PMP 70 among fetal tissues should be valid within a factor of 2 .

The middle panel of Figure 3 demonstrates clofibrate-induced increases in PMP 70, which are most striking in maternal liver (lane 2 versus 1 ) and kidney (lane 6 versus 5 ). Proportionally less PMP 70 is seen in maternal tissues such as brain (lanes 3 and 4) and lung (lanes 7 and 8 ), which contain microperoxisomes. A response to clofibrate is not evident in these tissues. The lower panel of Figure 3 demonstrates an increase in fetal liver PMP 70 both during ontogeny (lanes 5-8 representing gestational d 19-13) and as an effect of maternal clofibrate administration (lanes 1-4).

The average levels of PMP 70 in maternal tissue homogenates as measured in three separate experiments are shown in Figure 4 alongside sp act for the peroxisomal enzymes DHAP-AT and catalase, the microsomal enzyme GP-AT, the lysosomal enzyme acid phosphatase, and total protein. Figure 4 also summarizes the equivalent enzyme and protein measurements in 19-d fetal liver from clofibrate-treated or control pregnancies. Note that the enhancement of maternal and fetal peroxisomal proteins by clofibrate follows the order integral membrane protein (PMP 70) $>$ membrane-associated protein (DHAP-AT) $>$ matrix protein (catalase). Maternal kidney also shows some response to clofibrate and there is increased activity of DHAP-AT in the brains of treated mothers (Fig. 4). Clofibrate-treated livers are larger and yield more total protein in mothers, and this effect was noted for the liver and kidney of certain fetuses. Minimal differences in acid phosphatase or GP-AT activity were noted for maternal tissues or fetal liver (Fig. 4).

Figure 5 shows two sets of immunoblots contrasting the levels of PMP 70 in other fetal tissues from control or clofibrate-treated pregnancies. Those in Figure $5 A$ represent equivalent amounts of homogenate protein $(50 \mu \mathrm{g})$ from fetal brain $(b)$, lung $(l)$, kidney $(k)$, or placenta $(p)$ with those from control pregnancies in lanes 1-4 and from clofibrate-treated pregnancies in lanes 58 in order of decreasing gestational age (19-13 d). With these amounts of homogenate protein required to detect PMP 70 in fetal brain and lung, PMP 70 levels are close to saturation of the immunoblot assay in fetal kidney and placenta with minimal differences seen between clofibrate-treated and control pregnancies. Increased PMP 70 levels with ontogeny in fetal brain, lung, kidney (lanes 4-1) and with maternal clofibrate administration in fetal brain and lung (lanes 8-5) are demonstrated (Fig. 5A). In Figure $5 B$, only $20 \mu \mathrm{g}$ of each fetal tissue homogenate was applied to the gel with control (lanes $1-4$ ) and clofibrate-treated (lanes 5-8) in order of increasing gestational age (13-19 d). Dramatic effects of maternal clofibrate on fetal liver $(h)$ and placenta $(p)$ tissues are evident for 17- and 19-d samples, with a slight effect seen in fetal kidney $(k)$. The PMP 70 band is barely visible in fetal brain $(b)$ or lung $(l)$ when only $20 \mu \mathrm{g}$ of homogenate protein is applied to the gel (Fig. $5 B$ ). An increase of PMP 70 with normal ontogeny is also documented in fetal liver (Fig. 3 , bottom panel, lanes 8-5; Fig. 5B, lanes 1-4). Nonspecific 

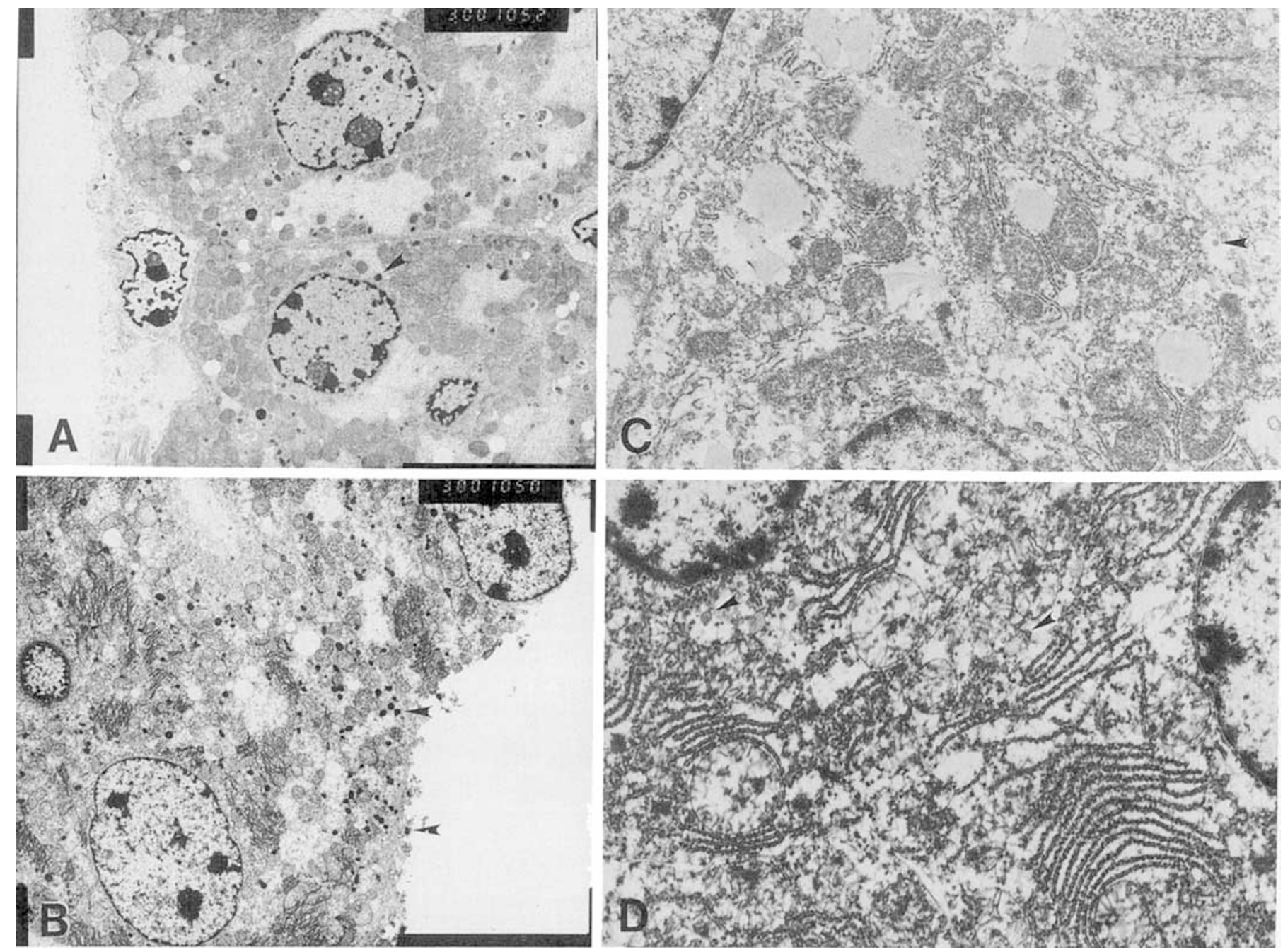

Fig. 2. Electron microscopy of control $(A)$ or clofibrate-treated $(B)$ maternal liver and control $(C)$ or clofibrate-treated $(D)$ 15-d fetal liver. Before fixation, the tissues were preincubated with diaminobenzidine, a catalase substrate that forms an electron-dense reaction product and enhances visualization of peroxisomes. Arrows illustrate peroxisomes; magnifications are $(A$ and $B) \times 1800,(C$ and $D) \times 2700$.

reaction of antibody to molecular weight markers (right lane) is seen in some blots depicted in Figure $5 A$ and $B$. This reaction probably reflects absorption to concentrated protein standards and has never been observed in experimental lanes.

The results from three separate breeding experiments are summarized in Figure 6, showing fetal tissue levels of peroxisomal or marker proteins as a function of maternal clofibrate administration. Enhancement of fetal PMP 70 by clofibrate occurs to some degree in all fetal tissues, but is most striking in fetal liver $(H)$ and kidney $(K)$. Enhancement of DHAP-AT sp act is also evident in all fetal tissues, and the effects of maternal clofibrate administration on these fetal peroxisomal membrane proteins can be seen by $13 \mathrm{~d}$ gestation in lung and placenta. Some variability of response in the three separate experiments summarized in Figure 6 is indicated by the standard error bars, but these were not calculated for PMP 70 because of the qualitative nature of the results. As with maternal and fetal liver (Fig. 4), the enhancement of membrane-associated proteins appears greater than that of the matrix protein catalase in most fetal tissues (Fig. 6). In fact, fetal lung and kidney catalase levels were actually higher in control pregnancies at certain stages of gestation. The amounts of peroxisomal proteins increase during normal ontogeny in all fetal tissues except perhaps in placenta. Maternal clofibrate increases total tissue protein only in fetal liver and kidney, the organs with the largest and most abundant peroxisomes (Fig. 6). No consistent differences in the sp act of acid phosphatase or GP-AT are seen in most fetal tissues, with the exception of a developmental decrease in acid phosphatase $\mathrm{sp}$ act in fetal kidney. There was a striking increase in the microsomal enzyme GP-AT in placentas from clofibrate-treated pregnancies (Fig. 6). As observed in human placenta (29), levels of mouse placenta peroxisomal proteins remain fairly constant in midgestation with a decrease in later pregnancy.

\section{DISCUSSION}

Several laboratories have demonstrated the presence of 150 -, $70 / 69-$, and $22-\mathrm{kD}$ PMP in rat $(28,30)$ or human $(11)$ tissues. Additional species of 53-, 42-, 36-, 28-, and 26-kD have also been seen in rat liver extracts, with the 42-, 28-, and 26-kD species postulated to be degradation products of the $70-\mathrm{kD}$ protein (30). No studies of mouse PMP have been reported, despite the potential in this organism to manipulate peroxisome structure through use of transgenic technology. We have detected PMP of 145-, 70-, 60-, 55-, 36-, and 22-kD PMP by protein staining or immunoblotting after SDS-PAGE of mouse liver homogenates (29). The immunoblot in Fig. $1 A$ demonstrates the 145-, 70-, 60-, and 55-kD peptides with the 36- and 22-kD peptides having run off the gel. The 145-, 70-, 36-, and 22-kD peptides appear highly conserved when liver homogenates of mouse, rat, and man are compared, and the variable detection of 60 - and $55-\mathrm{kD}$ peptides suggest that they are breakdown products of the 145- and 70-kD peptides (29). Rabbit antisera prepared against purified peroxisomal membranes from mouse 

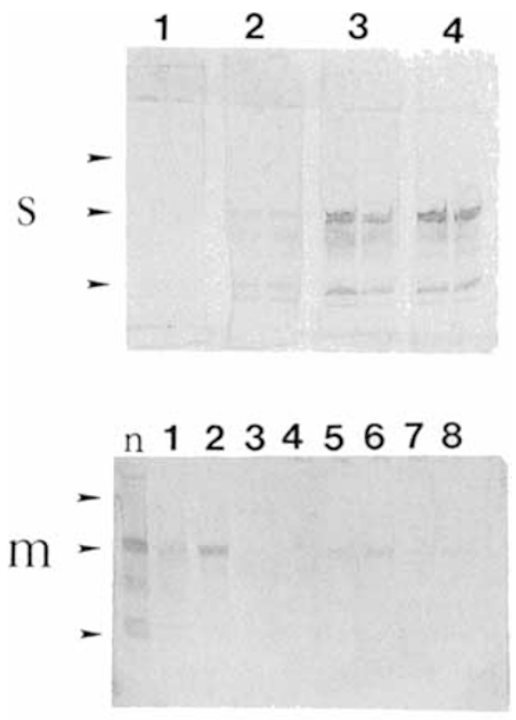

n12345678 s

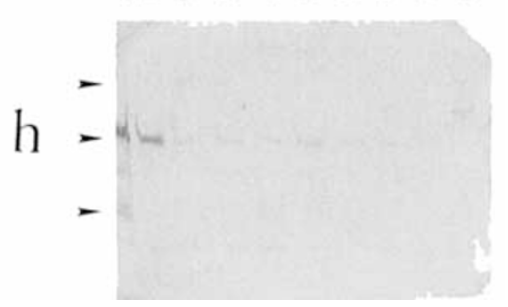

Fig. 3. The upper panel, $s$, shows a standard curve with duplicate lanes containing an estimated $0.05,0.1,0.2$, and $0.4 \mu \mathrm{g}$ of PMP 70 in purified peroxisomal membranes after detection with the standard immunoelectrophoresis assay. The middle panel, $m$, shows an immunoblot comparing $20 \mu \mathrm{g}$ of tissue homogenate: liver $(I, 2)$, brain $(3,4)$, kidney $(5,6)$, and lung $(7,8)$ from control $(I, 3,5,7)$ or 6-d clofibrate-treated $(2,4,6,8)$ adult females. The lower panel, $h$, shows an immunoblot comparing $20 \mu \mathrm{g}$ of liver homogenate from 19-, 17-, 15-, and 13-d fetuses of clofibrate-treated (1-4) or control (5-8) mothers. Lane $n$ is a mouse $\mathrm{N}_{\mathrm{G}}$ fraction control (19), and arrows indicate the positions of 145-, 70-, and $36-\mathrm{kD}$ peptides.

liver uniformly exhibit biased detection of the $70-\mathrm{kD}$ peptide, as illustrated in Figures $1 A, 3$, and 5 . Increased abundance, accessibility, or immunogenicity of this peptide relative to other PMP may be responsible. Although a conversion factor relating immunoblot band intensity to PMP 70 abundance cannot be reliably calculated, an estimate was made assuming equimolar ratios of PMP 145, 70, 36, and 22 in the peroxisomal membrane. Comparison to the standard curve in Figure 3 then allowed semiquantitative comparisons of PMP 70 levels among mater$\mathrm{nal} / \mathrm{fetal}$ tissue homogenates provided care was taken to ensure PMP 70 levels were not saturating for the immunoblot assay.

In agreement with a prior study in the rat $(17,18)$, the administration of clofibrate to pregnant mice is shown here to cause amplification of peroxisomal proteins in several fetal tissues. Whereas the rat experiments focused mainly on peroxisomal matrix proteins such as catalase or those responsible for fatty acid $\beta$-oxidation $(17,18)$, the data presented here demonstrate an effect on two other classes of peroxisomal proteins-integral membrane proteins (PMP 70) and the membrane-associated enzyme DHAP-AT - that is a key step in plasmalogen synthesis (9). Just as uncoupling of peroxisomal membrane and matrix synthesis is evident in certain disease states, the experiments summarized in Figures 4 and 6 show an increased clofibrate response of these membrane proteins relative to catalase. This response is consistent with initial synthesis of peroxisomal membranes and membrane proteins during biogenesis followed by

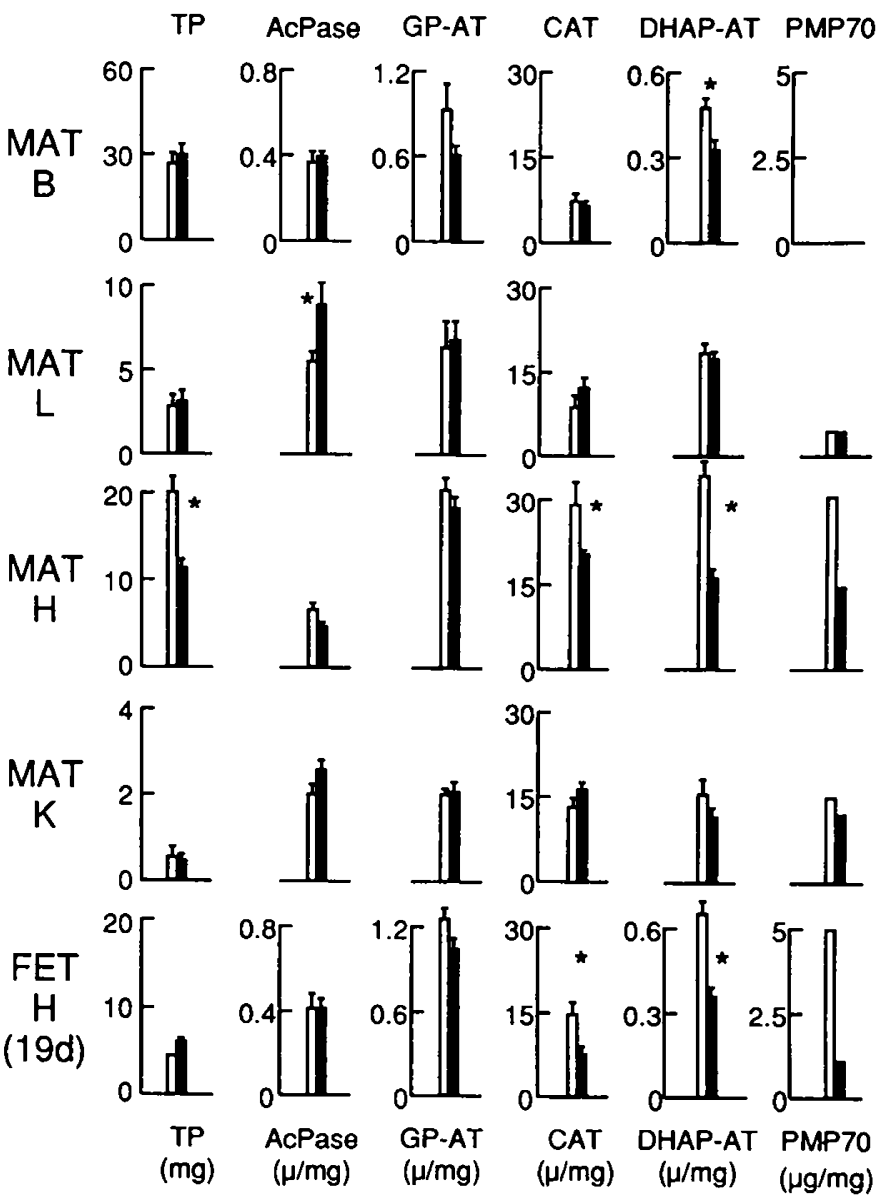

Fig. 4. Summary of clofibrate (empty bars) and control (filled bars) protein/enzyme levels in various maternal and 19-d fetal liver tissues. Levels of total protein, the lysosomal marker enzyme acid phosphatase (AcPase), the microsomal activity GP-AT, the matrix peroxisomal enzyme catalase $(C A T)$, the membrane-associated enzyme DHAP-AT, and the integral membrane protein PMP 70 are shown. Tissues are brain $(B)$, lung $(L)$, liver $(H)$, and kidney $(K)$. Standard errors are indicated above the bars except for PMP 70 levels, which were estimated by comparison to the standard curve in Figure 3. Significant differences $(p<0.05)$ are asterisked.

import of matrix components. Both PMP 70 and DHAP-AT were detected in most fetal tissues by $13 \mathrm{~d}$ of gestation, as was the matrix enzyme catalase. Because catalase can also be located in the cytoplasm, the detection of activity does not prove that a peroxisomal matrix has been assembled at this time in gestation. Failure to identify peroxisomes histologically early in gestation $(6,7)$ may reflect the primacy of peroxisomal membrane synthesis in both ontogeny and postnatal biogenesis. Consistent with this sequence is the decreased staining of fetal (Fig. $2 C$ and $D$ ) versus maternal (Fig. $2 A$ and $B$ ) liver peroxisomes with the diaminobenzidine reaction.

The ability to manipulate fetal peroxisomal membrane synthesis through administration of clofibrate to the mother is clearly demonstrated by the data summarized in Figures 4 and 6 . Fetal liver, lung, kidney, and placenta show evident increases in peroxisomal enzyme activities after initiating maternal clofibrate treatment at gestational $\mathrm{d} 6$. The response is most clear at 17-19 $\mathrm{d}$ gestation but may occur earlier inasmuch as early gestational expression in liver, as with later expression in brain or lung, is near the limits of detection for the immunoblot assay. The presence of peroxisomal components in mouse placenta early in gestation correlates with the demonstration of peroxisomes in human chorionic villi (8). More definitive evaluation of peroxisome biogenesis in the early mammalian embryo should soon be possible because a complementary DNA clone for the PMP 
b
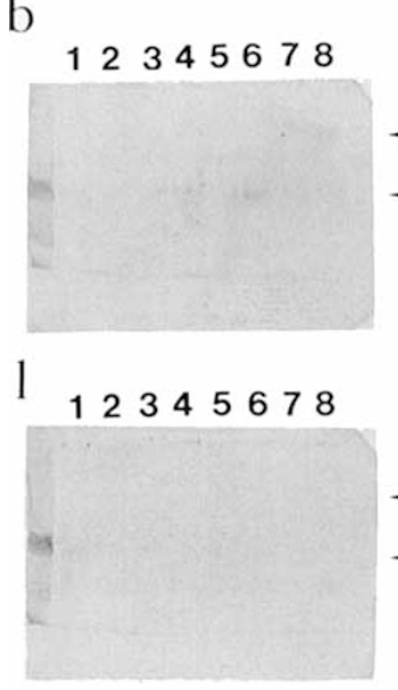

$\mathrm{p}$

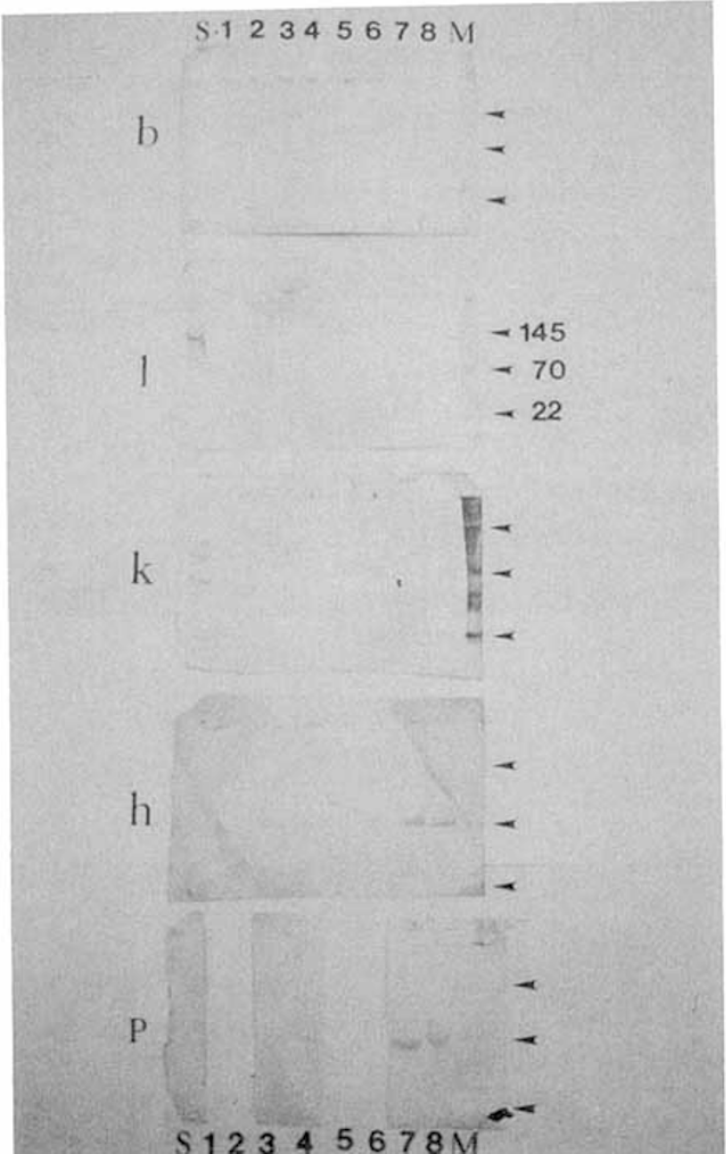

Fig. 5. Fetal tissue PMP 70 levels as a function of maternal clofibrate administration. $A$, Fetal brain $(b)$, lung $(l)$. placenta $(p)$, and kidney $(k)$ are shown at $19,17,15$, and $13 \mathrm{~d}$ of gestation with lanes $1-4$ from control and 5-8 from clofibrate-treated pregnancies. A mouse $\mathrm{N}_{\mathrm{G}}$ fraction is shown as control (left lane) with arrows indicating positions of the $145-$ and $70-\mathrm{kD}$ peptides. $B$, a separate experiment including fetal liver (h) with the order changed to 13-, 15-, 17-, and 19-d control (lanes 1-4) and clofibrate-treated (lanes 5-8) pregnancies. A mouse $N_{G}$ fraction (lane $M$ ) is included on blots $k$ and $p$ : arrows indicate positions of 145 -, 70-, and 36-kD PMP. Occasional nonspecific reaction of antibody with the molecular weight standards was detected in certain blots as shown in lane $S$.
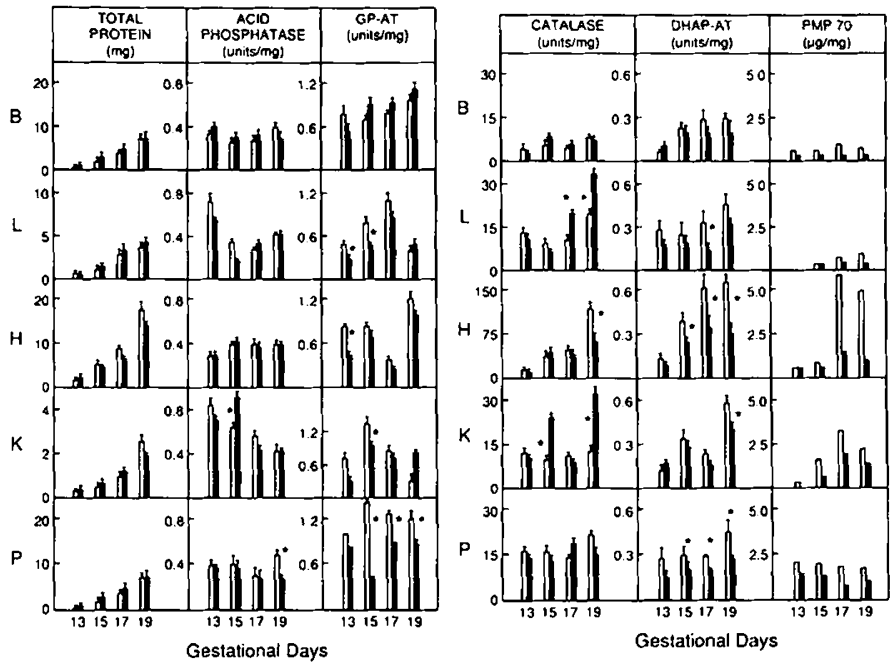

Fig. 6. Ontogeny of fetal proteins after maternal clofibrate (empty bars) or control (filled bars) treatment. Levels of total protein, acid phosphatase, and GP-AT are shown on the left, and those of catalase, DHAP-AT, and PMP 70 are shown on the right. Tissues are brain $(B)$, lung $(L)$, liver $(H)$, kidney $(K)$, and placenta $(P)$. Bars indicate standard error and asterisks significant differences $(p<0.05)$.

70 mRNA from rat liver has been isolated (32). Preliminary analysis of this clone demonstrates that it is a member of an ATP-binding protein family and supports the view that PMP are important structural components of the peroxisome.

Peroxisomal biogenesis during ontogeny, particularly because it can be regulated by proliferators such as clofibrate, offers a useful system for study of peroxisomal assembly. Peroxisomes were first thought to derive from the endoplasmic reticulum because electron micrographs may show connections between these organelles. The demonstration of unique polypeptides in peroxisomal membranes replaced this model with one asserting that new peroxisomes are formed by budding from previously existing peroxisomes (33). Peroxisomal fission is more a branching process than division, inasmuch as serial microscopy demonstrates a labyrinthine network of peroxisomal structure that appears circular only on cross-section (34). Integration of membrane proteins may occur by a different route than import of soluble proteins that are synthesized on free polysomes and targeted by carboxy-terminal or internal tripeptide signals (4, 34). The experimental system defined here provides the means to answer such questions through the combined effects of ontogeny and proliferative agents on a genetically manipulable organism.

\section{REFERENCES}

1. Santos MJ, Imanaka T, Shio H, Small GM, Lazarow PB 1988 Peroxisomal membrane ghosts in Zellweger syndrome-aberrant organelle assembly. Science 239:1536-1538

2. Baumgart E, VolkJ A, Hashimoto H, Fahimi HD 1989 Biogenesis of peroxisomes: immunocytochemical investigation of peroxisomal membrane proteins in proliferating rat liver peroxisomes and in catalase-negative membrane loops. J Cell Biol 108:2221-2231

3. Wilson GN. Holmes RD. Hajra AK 1988 Peroxisomal disorders: clinical commentary and future prospects. Am J Med Genet 30:771-792

4. Borst P 1989 Peroxisome biogenesis revisited. Biochim Biophys Acta 1008:113

5. Tsukada H, Mochizuki Y, Konishi T 1968 Morphogenesis and development of microbodies of hepatocytes of rats during pre- and postnatal growth. $J$ Cell Biol 37:231-243

6. Stefanini S, Farrace MG. Ceruargento MP 1985 Differentiation of liver peroxisomes in the fetal and newborn rat. Cytochemistry of catalase and Damino acid oxidase. J Embryol Exp Morphol 88:151-163

7. Briere N 1986 Peroxisomes in human fetal kidncy: variations in size and number during development. Anat Embryol (Berl) 174:235-242

8. Roels F, Verdonck V, Pauwels M, De Catte L, Lissens W, Liebaers 1, Elleder M 1987 Light microscopic visualization of peroxisomes and plasmalogens in first trimester chorionic villi. Prenat Diagn 7:525-530 
9. Burke CL, Hajra AK 1980 Clofibrate feeding increases the activities of rat liver dihydroxyacetone phosphate acyltransferase and glycerol-3-phosphate acyltransferase. Biochem Int 1:312-318

10. Rao MS, Reddy JK 1987 Peroxisome proliferation and hepatocarcinogenesis. Carcinogenesis 8:631-636

11. Veerkamp JH, van Moerkerk HTB 1986 Peroxisomal fatty acid oxidation in rat and human tissues. Effect of nutritional state, clofibrate treatment and postnatal development in the rat. Biochim Biophys Acta 875:301-310

12. Lalwani ND, Reddy K, Ghosh S, Barnard SD, Molello JA, Reddy JK 1985 Induction of fatty acid $\beta$-oxidation and peroxisome proliferation in the liver of rhesus monkeys by DL-040, a new hypolipidemic agent. Biochem Pharmacol 34:3473-3482

13. Van den Branden C, Vamecq J, Wybo I, Roels F 1986 Phytol and peroxisome proliferation. Pediatr Res 20:411-4is

14. Lazarow PB, Black V, Shio H, Fujiki Y, Hajra AK, Datta NS, Bangaru BS Dancis J 1985 Zellweger syndrome: biochemical and morphological studies on two patients treated with clofibrate. Pediatr Res 19:1356-1364

15. Reddy JK, Goel SK, Nemali MR, Carrino JJ, Laffler TG, Reddy MK, Sperbeck SH, Osumi T, Hashimoto T, Lalwani ND, Rao MS 1986 Transcriptional regulation of peroxisomal fatty acyl-CoA oxidase and enoyl-CoA hydratase/ 3-hydroxy-CoA dehydrogenase in rat liver by peroxisome proliferators. Proc Natl Acad Sci USA 83:1747-1751

16. Milton MN, Elcombe CR, Kass GEN, Gibson GG 1988 Lack of evidence for a hepatic peroxisome proliferator and an explanation for the binding of hypolipidaemic drugs to liver homogenates. Biochem Pharmacol 37:793798

17. Cibelli A, Stefanini S, Ceru MP 1988 Peroxisomal $\beta$-oxidation and catalase activities in fetal rat liver: effect of maternal treatment with clofibrate. Cell Mol Biol 34:191-205

18. Stefanini S, Mauriello A, Farrace MG, Cibelli A, Ceru MP 1989 Proliferative response of foetal liver peroxisomes to clofibrate treatment of pregnant rats. A quantitative evaluation. Biol Cell 67:299-305

19. Peters TJ, Muller M, De Duve C 1972 Lysosomes of the arterial wall. I Isolation and subcellular fractionation of cells from normal rabbit aorta. J Exp Med 136:1117-1123

20. Jones CL, Hajra AK 1980 Properties of guinea pig liver peroxisomal dihydroxyacetone phosphate acyltransferase. J Biol Chem 255:8289-8295

21. Datta NS, Wilson GN, Hajra AK 1984 Deficiency of enzymes catalyzing the biosynthesis of glycerol-ether lipids in Zellweger syndrome. N Engl J Med 311:1080-1083

22. Leighton F, Poole B, Beaufay H, Baudhuin P, Coffey JW, Fowler S, De Duve C 1968 The large-scale separation of peroxisomes, mitochondria, and lysosomes from the livers of rats injected with Triton WR-1339. J Cell Biol 37:482-513

23. Racker E 1966 Spectrophotometric measurement of hexokinase and phosphohexokinase activity. Methods Enzymol 1:843-846

24. Bradford MM $1976 \mathrm{~A}$ rapid and sensitive method for the quantitation of microgram quantities of protein using the principle of protein-dye binding. Anal Biochem 72:248-254

25. Lowry OH, Rosebrough NJ, Fart AL, Randall RJ 1951 Protein measurement with the Folin phenol reagent. J Biol Chem 193:265-275

26. Fujiki Y, Fowler S, Shio H, Hubbard AL, Lazarow PB 1982 Polypeptide and phospholipid composition of the membrane of rat liver peroxisomes: comparison with endoplasmic reticulum and mitochondrial membranes. J Cell Biol 93:103-110

27. Ghosh MK, Hajra AK 1986 A rapid method for the isolation of peroxisomes from rat liver. Anal Biochem 159:169-174

28. Santos MJ, Imanaka T, Shio H, Small GM, Lazarow PB 1988 Peroxisoma integral membrane proteins in control and Zellweger fibroblasts. J Biol Chem 263:10502-10509

29. Wilson GN, Argyle JC, King T 1990 Maternal clofibrate administration amplifies fetal peroxisomes. Pediatr Res 27:195A(abstr)

30. Hartl F-U, Just WW 1987 Integral membrane polypeptides of rat liver peroxisomes: topology and response to different metabolic states. Arch Biochem Biophys 255:109-119

31. Small GM, Santos MJ, Imanaka T, Poulos A, Danks DM, Moser HW, Lazarow PB 1988 Peroxisomal integral membrane proteins in livers of patients with Zellweger syndrome, infantile Refsum's disease and X-linked adrenoleukodystrophy. J Inherited Metab Dis 11:358-371

32. Kamijo K, Taketani S, Yokota S, Osumi T, Hashimoto T 1990 The 70-kDa peroxisomal membrane protein is a member of the $\mathrm{Mdr}$ (P-glycoprotein)related ATP-binding protein superfamily. J Biol Chem 265:4534-4540

33. Lazarow PB, Fujiki Y 1985 Biogenesis of peroxisomes. Annu Rev Cell Biol $1: 489-530$

34. Yamamoto K, Fahimi HD 1987 Three-dimensional reconstruction of a peroxisomal reticulum in regenerating rat liver: evidence of interconnections between heterogeneous segments. J Cell Biol 105:713-722 